\title{
Numerical Solution of Inviscid Compressible Steady Flows around the RAE 2822 Airfoil
}

\author{
P. Kryštůfek ${ }^{1, \text { a }}$, K. Kozel ${ }^{2}$ \\ ${ }^{1}$ Technical University of Liberec, Faculty of Mechanical Engineering, Czech Republic \\ ${ }^{2}$ Czech Technical University in Prague, Faculty of Mechanical Engineering, Czech Republic
}

\begin{abstract}
The article presents results of a numerical solution of subsonic, transonic and supersonic flows described by the system of Euler equations in 2D compressible flows around the RAE 2822 airfoil. Authors used FVM multistage Runge-Kutta method to numerically solve the flows around the RAE 2822 airfoil. The results are compared with the solution using the software Ansys Fluent 15.0.7.
\end{abstract}

\section{Introduction}

Numerical Solution of Inviscid Compressible Steady Flows around the profile also dealt with in 1983 at a conference in France Antony Jameson [1]. His results were for profiles NACA 64A410, NACA 0012 and Korn. James used multistage Runge-Kutta numerical scheme on non-orthogonal structured grids with $160 \times 32$ elements. Furthermore, this problem also dealt paper [2]. Numerical calculations were performed on a profile DCA $18 \%$ by using a multistage Runge-Kutta numerical scheme on non-orthogonal structured grids with $160 \times 160$ elements.

The article follows the previous successful results [67] and contains a numerical code for simulating subsonic, transonic and supersonic flows field around the RAE 2822 airfoil. In these simulations the meshes type C has been used, which was created by mesh generator and has been described in [8-9]. In this case mesh has been created for numerical solution over profile RAE 2822.

For comparison of the results was used commercial software Ansys Fluent 15.0.7, which was simulated the same problem. In this case mesh has been created in Ansys Meshing.

\section{Mathematical models}

The 2D flow of an inviscid compressible fluid is described by the system of Euler equations

$$
\mathbf{W}_{t}+\mathbf{F}_{x}+\mathbf{G}_{y}=0
$$

$$
\begin{aligned}
& \mathbf{W}=\left(\rho, \rho w_{1}, \rho w_{2}, e\right)^{T}, \\
& \mathbf{F}=\left(\rho w_{1}, \rho w_{1}^{2}+p, \rho w_{1} w_{2},(e+p) w_{1}\right)^{T}, \\
& \mathbf{G}=\left(\rho w_{2}, \rho w_{1} w_{2}, \rho w_{2}{ }^{2}+p,(e+p) w_{2}\right)^{T},
\end{aligned}
$$

and [4]

$$
p=(\kappa-1)\left[e-\frac{1}{2}\left(w_{1}^{2}+w_{2}^{2}\right)\right]
$$

In the above equations, $\mathbf{W}$ is conservative variable, F, $\mathbf{G}$ are function of inviscid physical fluxes, $\rho$ denotes density, $w_{1}, w_{2}$ are components of velocity in the direction of axis $x, y ; p$ is pressure, $e$ is total energy per unit volume. The parameter $\kappa=1,4$ is the adiabatic exponent.

All variables were considered dimensionless and inflow variables (with the suffix $\infty$ ) were used as the reference variable for the transformation of the equations to dimensionless form.

\section{Numerical methods}

For modeling of the mentioned flow case, numerical scheme of FVM multistage Runge-Kutta (RK) is used on non-orthogonal structured grids of quadrilateral cells $D_{i j}$.

where

${ }^{a}$ e-mail: pavel.krystufek@tul.cz 


$$
\begin{aligned}
& \mathbf{W}_{i, j}^{(0)}=\mathbf{W}_{i, j}^{(n)}, \\
& \mathbf{W}_{i, j}^{(r+1)}=\mathbf{W}_{i, j}^{(0)}-\Delta t \cdot \alpha_{r} \cdot \operatorname{Rez} \mathbf{W}_{i, j}^{(r)}+A D\left(\mathbf{W}_{i, j}^{(r)}\right), \\
& \mathbf{W}_{i, j}^{(n+1)}=\mathbf{W}_{i, j}^{(m)}, \\
& \operatorname{Rez}_{i, j}^{(n)}=\frac{1}{\left|D_{i, j}\right|} \sum_{k=1}^{4}\left[\tilde{\mathbf{F}}_{k}^{n} \Delta y_{k}-\tilde{\mathbf{G}}_{k}^{n} \Delta x_{k}\right],
\end{aligned}
$$

for

$$
\begin{gathered}
r=0,1, \ldots, m-1 ; m=5 \\
\alpha_{1}=0,25 ; \alpha_{2}=0,1667 ; \alpha_{3}=0,375 ; \alpha_{4}=0,5 ; \alpha_{5}=1
\end{gathered}
$$

where $\left|D_{i, j}\right|$ represents the surface or volume of the cell. Numerical approximations $\tilde{\mathbf{F}}, \tilde{\mathbf{G}}$ of convective terms $\mathbf{F}, \mathbf{G}$ were considered in the forward form of the first order of accuracy.

The scheme was extended to include Jameson's artificial dissipation in (9) because of the stability of the method

$$
\begin{aligned}
A D\left(\mathbf{W}_{i, j}^{(n)}\right)= & k_{1} \cdot \gamma_{i} \cdot\left(\mathbf{W}_{i+1, j}^{(n)}-2 \mathbf{W}_{i, j}^{(n)}+\mathbf{W}_{i-1, j}^{(n)}\right) \\
& +k_{2} \cdot \gamma_{j} \cdot\left(\mathbf{W}_{i, j+1}^{(n)}-2 \mathbf{W}_{i, j}^{(n)}+\mathbf{W}_{i, j-1}^{(n)}\right),
\end{aligned}
$$

where

$$
\begin{aligned}
& \gamma_{i}=\frac{\left|p_{i+1, j}^{n}-2 p_{i, j}^{n}+p_{i-1, j}^{n}\right|}{\left|p_{i+1, j}^{n}\right|+2\left|p_{i, j}^{n}\right|+\left|p_{i-1, j}^{n}\right|}, \\
& \gamma_{j}=\frac{\left|p_{i, j+1}^{n}-2 p_{i, j}^{n}+p_{i, j-1}^{n}\right|}{\left|p_{i, j+1}^{n}\right|+2\left|p_{i, j}^{n}\right|+\left|p_{i, j-1}^{n}\right|} .
\end{aligned}
$$

The convergence to the steady state is followed by log L2 residual defined by

$$
\operatorname{Rez}_{i, j}^{(n)}=\frac{1}{N} \sqrt{\sum_{i, j}\left(\frac{\mathbf{W}_{i, j}^{(n+1)}-\mathbf{W}_{i, j}^{(n)}}{\Delta t}\right)^{2}}
$$

where $\mathrm{N}$ is a number of all elements in the computational domain.

\section{Specification of test case}

We used RAE 2822 airfoil. This airfoil was presented in figure 1 .

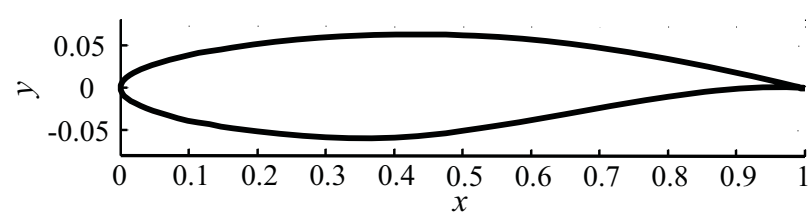

Figure 1. RAE 2822 airfoil (by Kryštůfek).

The outlines of the computational domain are shown in figure 2. In this computational domain was placed RAE 2822 airfoil.

We selected for numerical solution a structured mesh formed by quadrilateral finite volumes. The grid around profiles (wing) usually consists of a $\mathrm{C}$-grid in the flow direction. In the case of the C-topology the aerodynamics body is enclosed by one family grid lines, which also form the wake region.

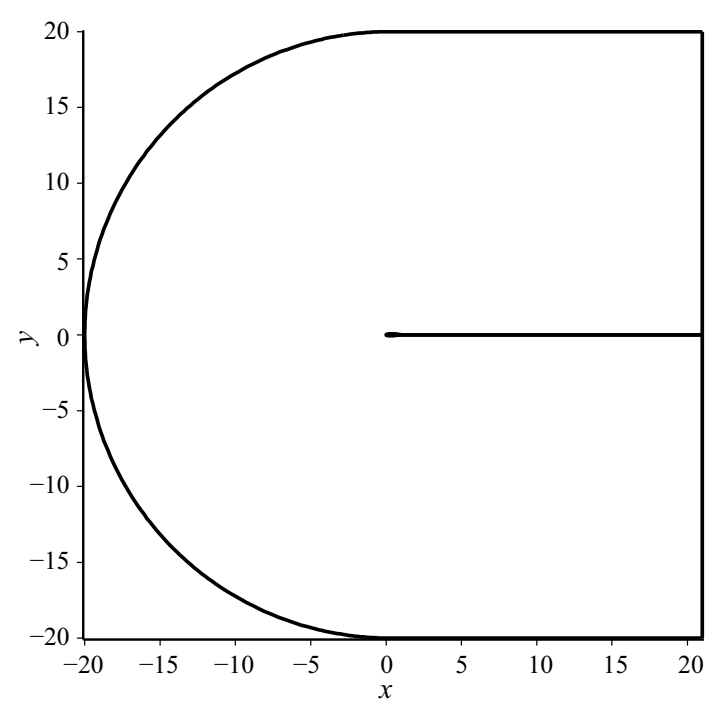

Figure 2. Computational domain.

The RAE 2822 airfoil contour is approximated by a Bezier spline [8-9]. The grid with $384 \times 64$ elements created by ours program for this simulation were presented in figure 3 .

The second grid was created in Ansys Meshing. Details of this grid with 1 million elements are shown in figures 4 and 5 . We also used structured mesh formed by quadrilateral finite volumes.

\subsection{Boundary conditions}

For supersonic inflow the conservative variables on the boundary are determined by freestream values only. The values are specified based on the given Mach number $M a_{\infty}$ and two flow angles (angle of attack and side-slip angle). 


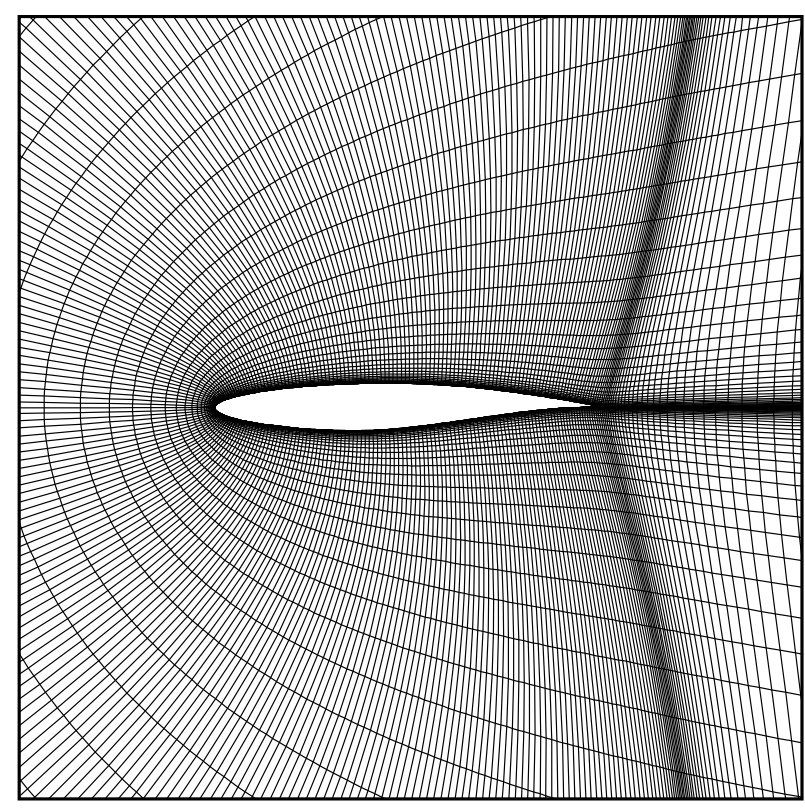

Figure 3. C-type, RAE 2822 airfoil, 384x64 elements (by Kryštůfek [7]).

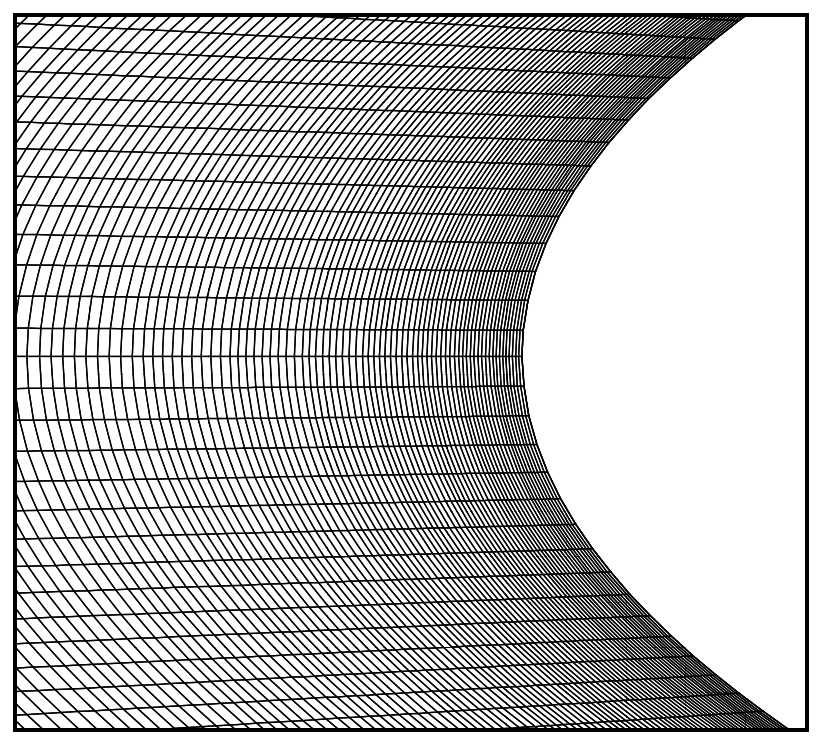

Figure 4. Grid generated by Ansys Meshing, RAE 2822 airfoil, 1 million elements, leading part of the profile.

For supersonic outflow all conservative variables at the boundary must be determined from the solution inside domain.

Three characteristic variables are prescribed based on the free stream values at far field subsonic inflow. One characteristic variable is extrapolated from interior of the physical domain.

In the case of subsonic outflow, three flow variables (density and the two velocity components) have to be extrapolated from interior of the physical domain. The remaining four variables (pressure) must be specified externally (as a multiple of the input pressure).

On wall zero derivatives of velocity vector along normal is considered. This condition is implemented by using virtual cells. Such cells adjoin from outside on the boundary cells and we prescribe values of unknowns inside of them to obtain the desired effect.

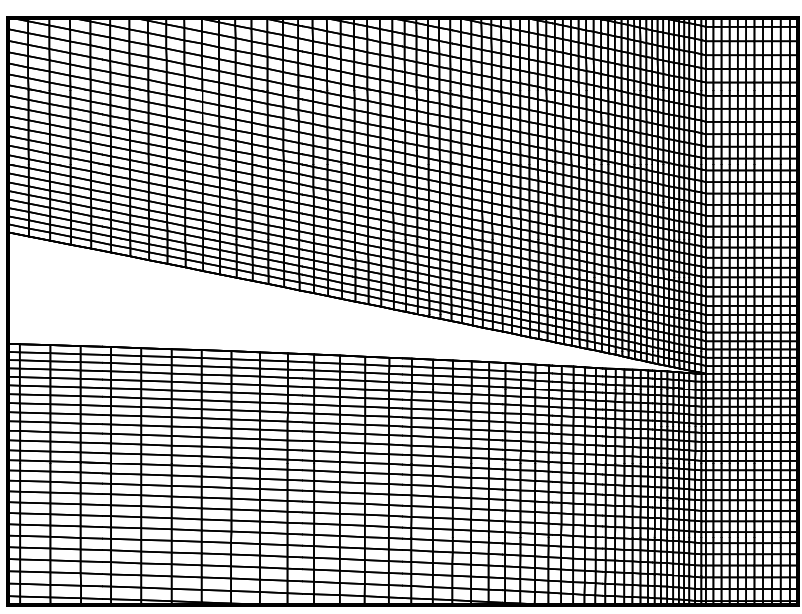

Figure 5. Grid generated by Ansys Meshing, RAE 2822 airfoil, 1 million elements, end of the profile.

\section{Numerical results and conclusion}

The authors have dealt with the numerical simulation of 2D laminar transonic flows around the RAE 2822 airfoil and applied the multistage Runge-Kutta method and orthogonal structured grids C type with $384 \times 64$ cells for its created by the linear TFI method [5]. The authors considered a few different values of inlet Mach number $M a_{\infty} \in(0.675,1.1)$ and angle of attack $\alpha=0^{\circ}$. The numerical results are presented in Mach number isolines and Mach number on profile.

On figures 6, 7, 8 are shown Mach number isolines for inviscid compressible flow around RAE 2822 airfoil. On figures 9, 10, 11 are shown Mach number isolines for same test case created in software Ansys Fluent 15.0.07. The results are in good agreement.

For more detailed analysis of results, the authors view the Mach number at the top and bottom profile depending on the length of the profile. On figures 12, 13, 14 are shown the progress of Mach number on the profile for $M a_{\infty}=0.675, M a_{\infty}=0.775$ and $M a_{\infty}=1.1$. Progress of Mach numbers on the top side of the profile are shown on the top figure and progress of Mach numbers on the bottom side of the profile are shown on the lower figure. On them are compared our results with the results from the Ansys Fluent 15.0.7.

For subsonic flow ( $\left.M a_{\infty}=0.675\right)$ is good agreement curves of Mach numbers for the bottom side of the profile. For the top side of the profile are distinct values greater of Mach number along the entire length of the profile, especially at the beginning. At the end of the profile is to see a larger decrease (hollow) of Mach number by our progress.

For transonic flow $\left(M a_{\infty}=0.775\right)$ is good agreement curves of Mach numbers for both parts of the profile. Positions of the shock waves are in good agreement. Values of the Mach numbers from Ansys 15.0.7 are slightly smaller at the beginning of the profile. 


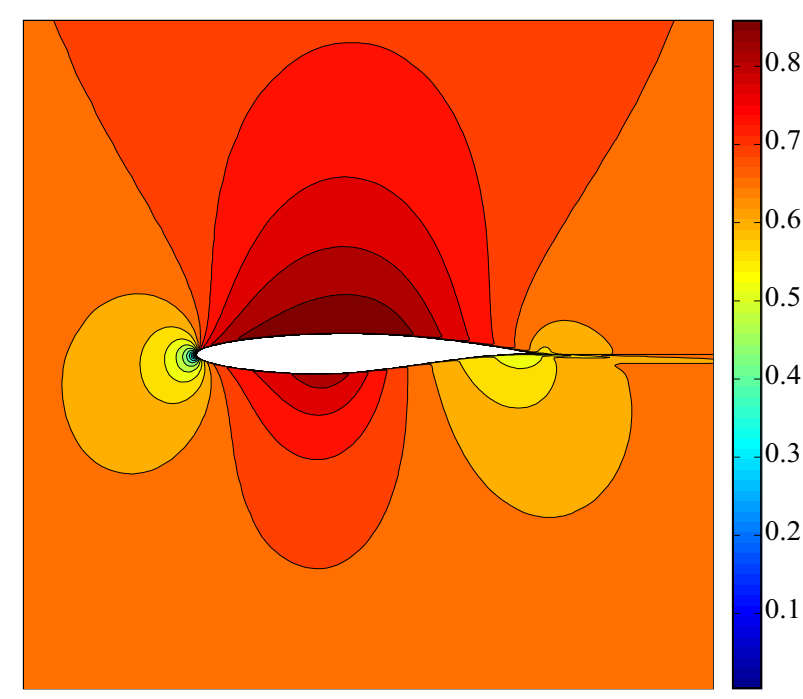

Figure 6. Inviscid compressible flow around RAE 2822 airfoil, C-type mesh with 384x64 cells, Mach number isolines - RK scheme, $M a_{\infty}=0.675$ and $\alpha=0^{\circ}$.

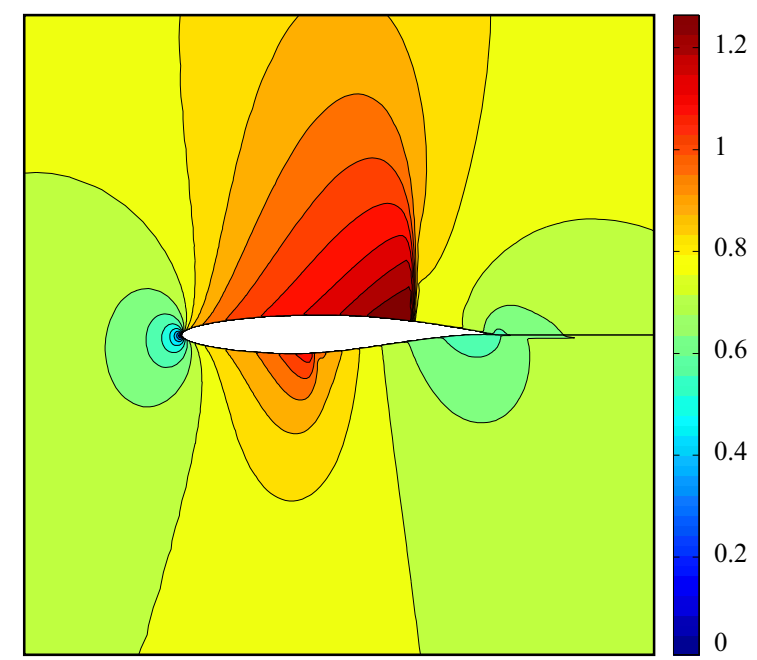

Figure 7. Inviscid compressible flow around RAE 2822 airfoil, C-type mesch with $384 \times 64$ cells, Mach number isolines - RK scheme, $M a_{\infty}=0.775$ and $\alpha=0^{\circ}$.

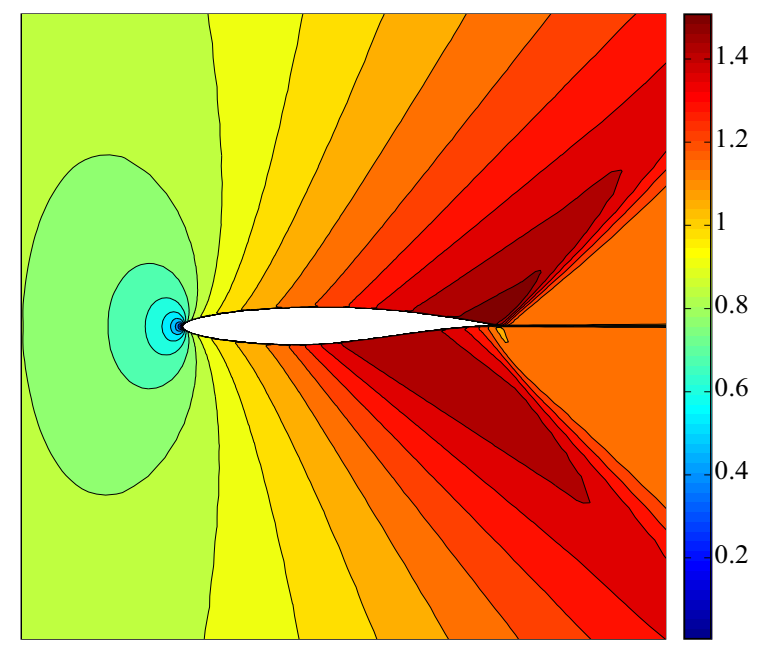

Figure 8. Inviscid compressible flow around RAE 2822 airfoil, C-type mesh with 384x64 cells, Mach number isolines - RK scheme, $M a_{\infty}=1.1$ and $\alpha=0^{\circ}$.

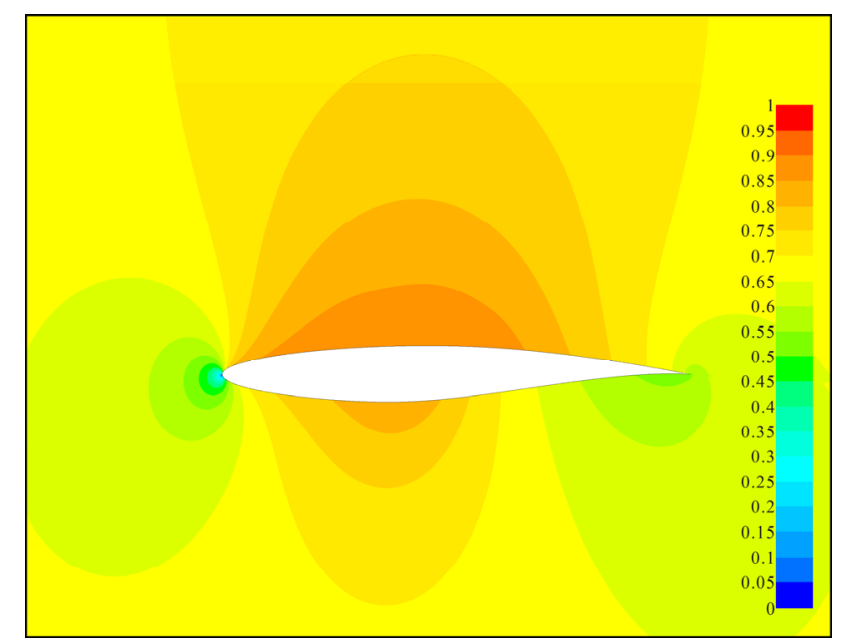

Figure 9. Inviscid compressible flow around RAE 2822 airfoil, Mach number isolines, Ansys Fluent 15.0.7, 1 million elements, $M a_{\infty}=0.675, \alpha=0^{\circ}$.

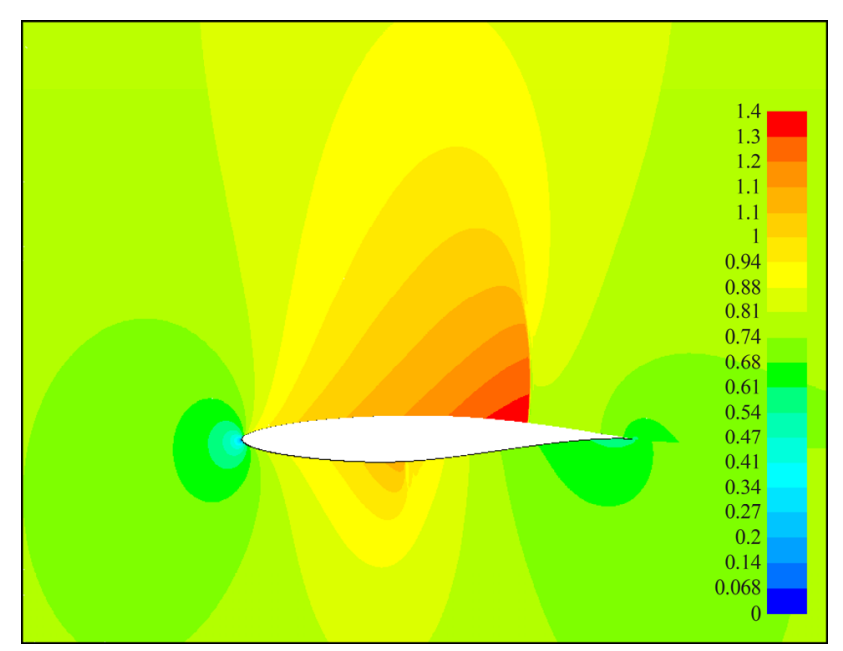

Figure 10. Inviscid compressible flow around RAE 2822 airfoil, Mach number isolines, Ansys Fluent 15.0.7, 1 million elements, $M a_{\infty}=0.775, \alpha=0^{\circ}$.

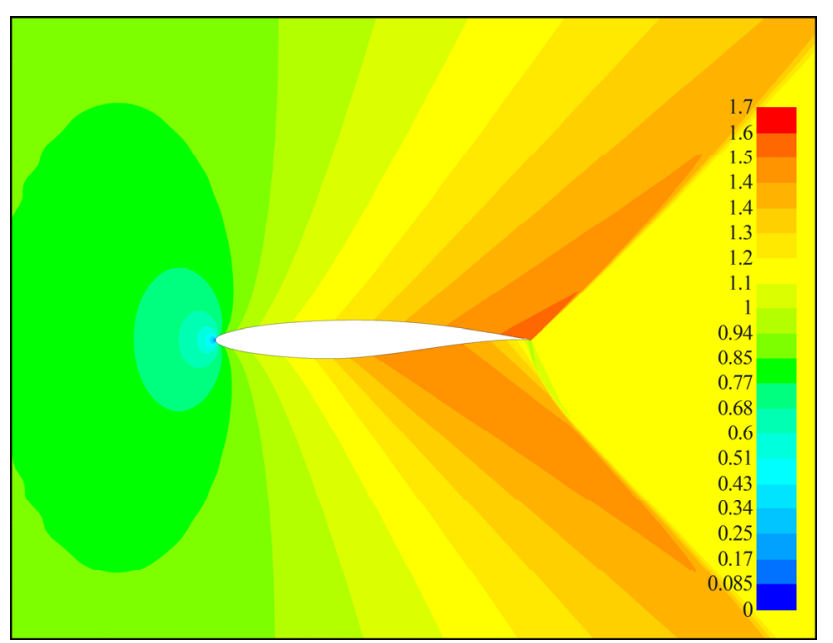

Figure 11. Inviscid compressible flow around RAE 2822 airfoil, Mach number isolines, Ansys Fluent 15.0.7, 1 million elements, $M a_{\infty}=1.1, \alpha=0^{\circ}$. 

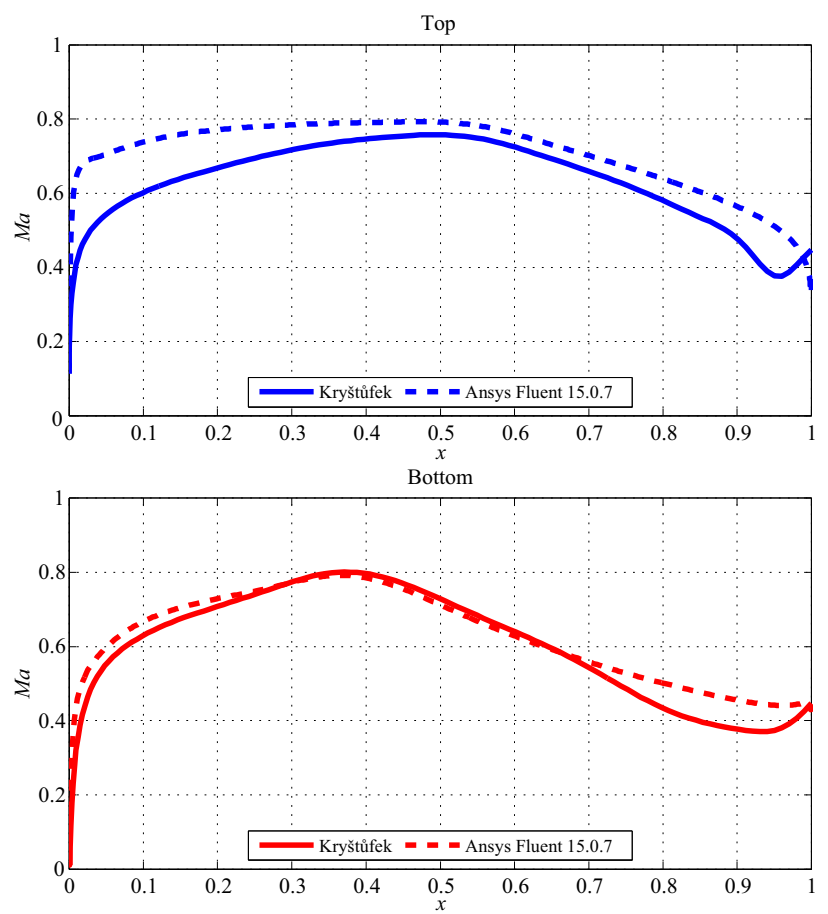

Figure 12. Progress of Mach numbers on the top side and bottom side of the profile for $M a_{\infty}=0.675, \alpha=0^{\circ}$.
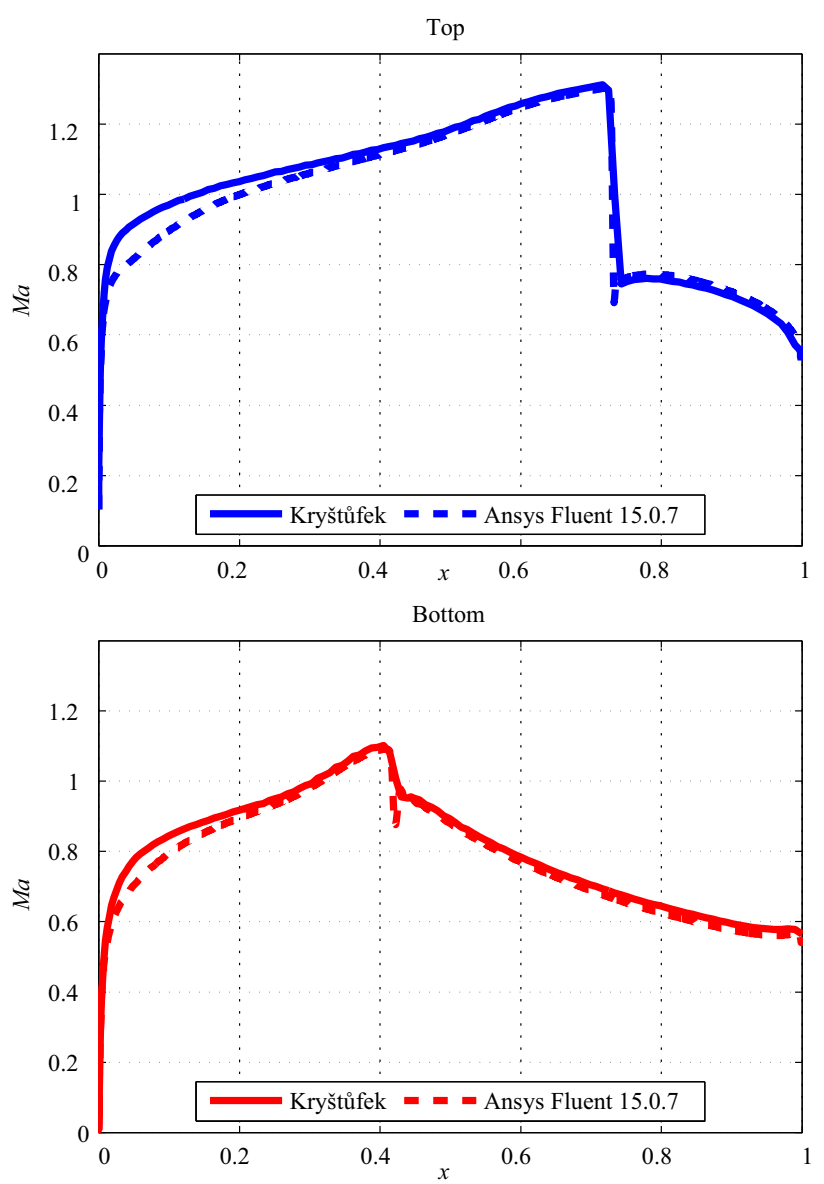

Figure 13. Progress of Mach numbers on the top side and bottom side of the profile for $M a_{\infty}=0.775, \alpha=0^{\circ}$.
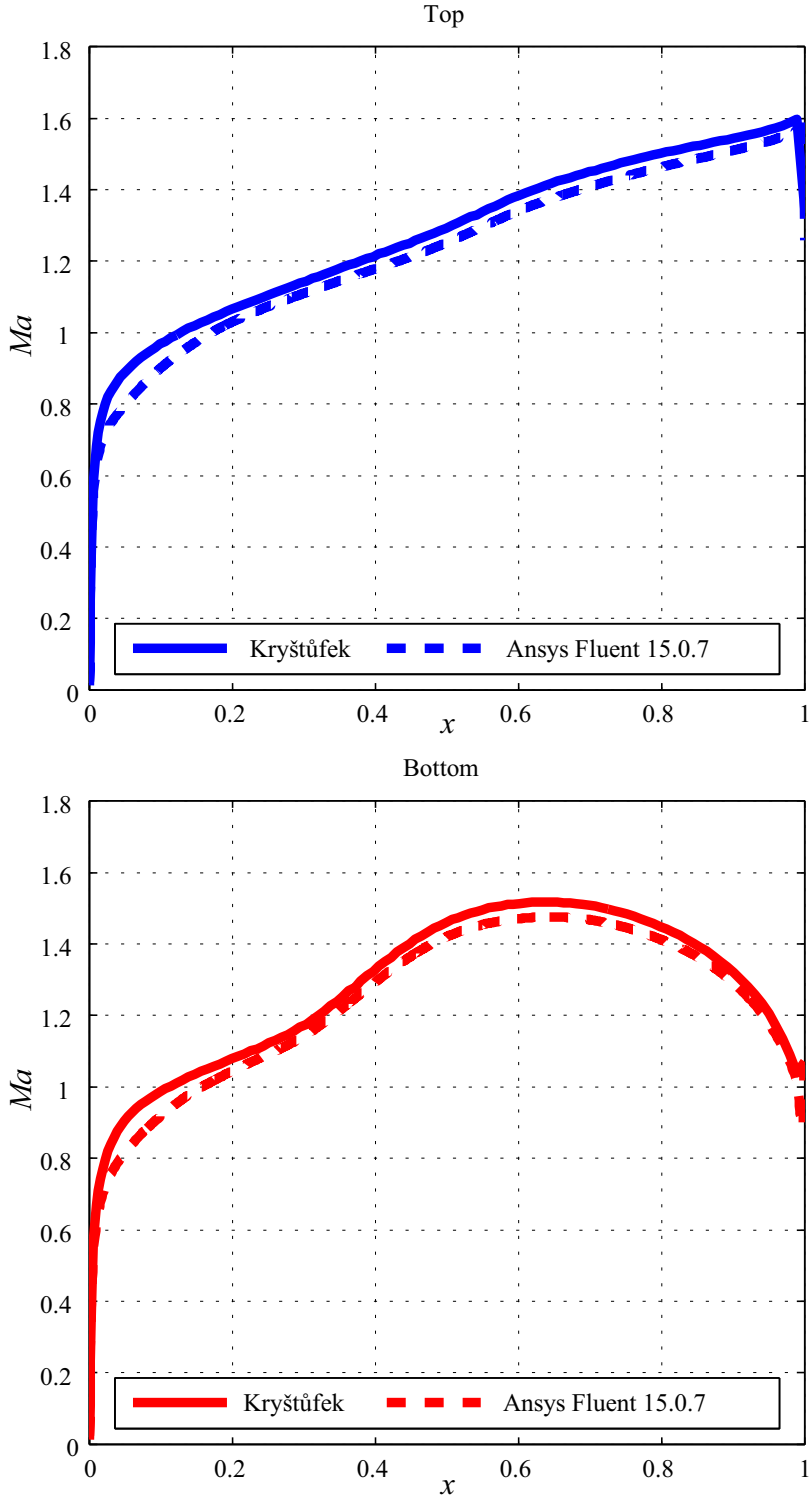

Figure 14. Progress of Mach numbers on the top side and bottom side of the profile for $M a_{\infty}=1.1, \alpha=0^{\circ}$.

For supersonic flow $\left(M a_{\infty}=1.1\right)$ is very good agreement curves of Mach numbers for both parts of the profile. The shockwave is visible only on the top side of the profile and in both cases in the same position on the profile. Values from Ansys 15.0.7 are slightly smaller along the entire length of profile, but very well follow the shape of our results.

On figure 15 is shown convergence of residuals on iterations for $M a_{\infty}=0.675$. On figure 16 is shown convergence of residuals on iterations for $M a_{\infty}=0.675$ for solution in Ansys Fluent 15.0.7. It is seen that in both solvers is convergence of residuals continuous.

The results can be improved by suitable choice of parameters of Jameson's artificial dissipation. In the future, we will continue with an algebraic turbulence model and turbulence two-equation model. 


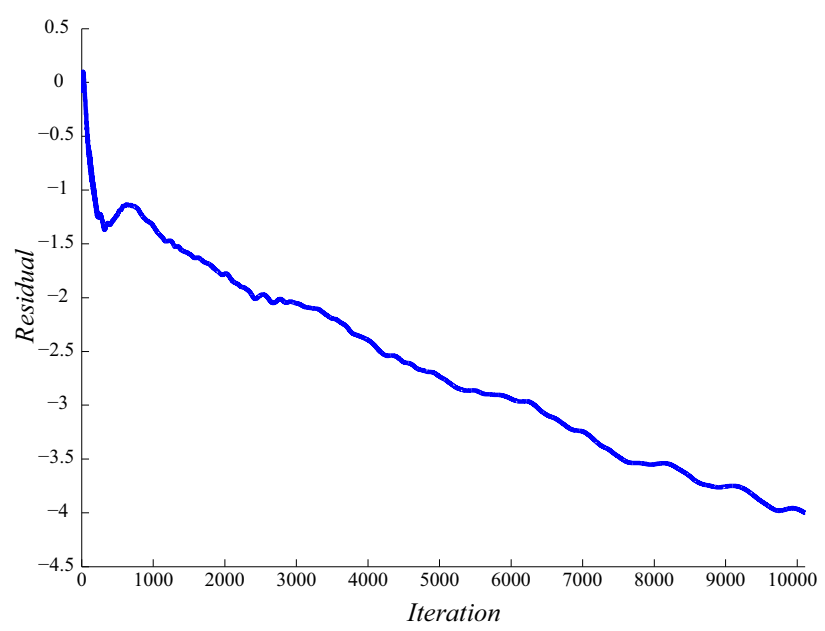

Figure 15. Residuals for $M a_{\infty}=0.675, \alpha=0^{\circ}$.

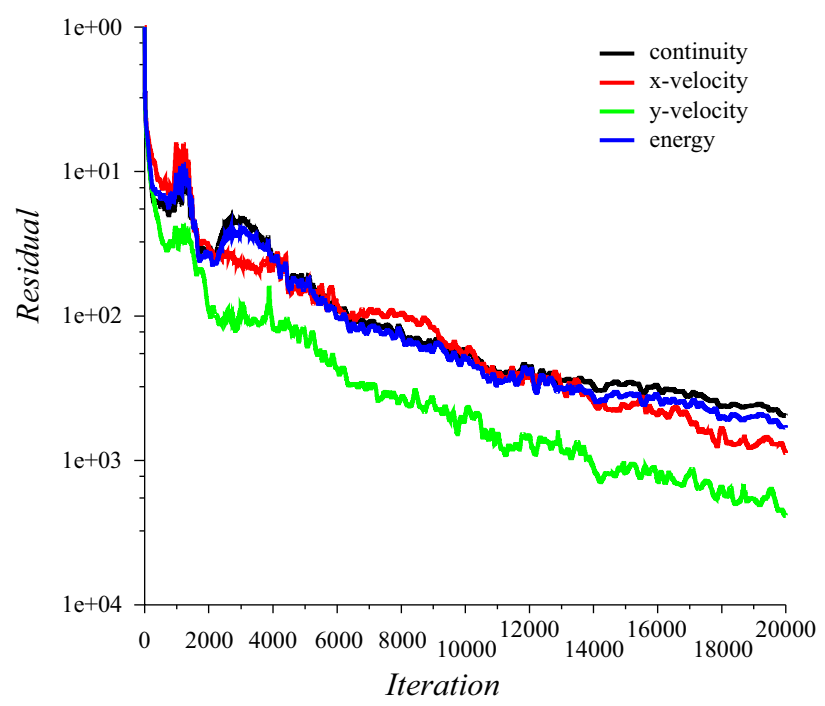

Figure 16. Residuals for $M a_{\infty}=0.675, \alpha=0^{\circ}$,

Ansys Fluent 15.0.7.

\section{Acknowledgements}

This work was financially supported by the particular research student grant SGS 28000 at TU of Liberec and GAČR P101/11/0207, GAČR P101/10/1329.

\section{References}

1. A. Jameson, Euler Workshop (1983)

2. J. Huml, K. Kozel, Experimental fluid mechanics, 295-299 (2013)

3. A.J.W. Sommerfeld, Partial Differential Equations in Physics (1949)

4. F. M. White, Fluid Mechanics - Fourth Edition (2001)

5. J. Blazek, Computational Fluid Dynamics: Principles and Applications (2005)

6. P. Kryštůfek, K. Kozel, Experimental fluid mechanics, 390-393 (2013)

7. P. Kryštůfek, K. Kozel, Experimental fluid mechanics, 371-374 (2012)
8. P. Kryštůfek, K. Kozel, Experimental fluid mechanics, 771-777 (2011)

9. P. Kryštůfek, K. Kozel, Power System Engineering, Thermodynamics \& Fluid Flow (2012) 